\title{
The magnetic properties of potassium holmium double tungstate
}

\author{
M.T. Borowiec ${ }^{1}$, V.P. Dyakonov ${ }^{1,2}$, E.N. Khatsko ${ }^{1,3}$, T. Zayarnyuk ${ }^{1}$, E.E. Zubov ${ }^{1,2}$, \\ A. Szewczyk ${ }^{1}$, M.U. Gutowska ${ }^{1}$, A.I. Rykova ${ }^{3}$, J. Piętosa ${ }^{1}$, A. Majchrowski $^{4}$, \\ E. Michalski ${ }^{4}$, J.-U. Hoffmann ${ }^{5}$, K. Prokes ${ }^{5}$, K. Woźniak ${ }^{6}$, Ł. Dobrzycki ${ }^{6}$, M. Barański ${ }^{1}$, \\ V. Domukhovski ${ }^{1}$, V. Shtyrkhunova ${ }^{2}$, J. Żmija ${ }^{4}$, and H. Szymczak ${ }^{1}$ \\ ${ }^{1}$ Institute of Physics, Polish Academy of Sciences, 32/46 Al. Lotników, Warsaw 02 668, Poland \\ E-mail: borow@ifpan.edu.pl \\ ${ }^{2}$ O.O. Galkin Donetsk Institute for Physics and Engineering of the National Academy of Sciences of Ukraine \\ 72 R. Luxembourg Str., Donetsk 83114, Ukraine \\ ${ }^{3}$ B. Verkin Institute for Low Temperature Physics and Engineering of the National Academy of Sciences of Ukraine \\ 47 Lenin Ave., Kharkov 61103, Ukraine \\ ${ }^{4}$ Institute of Applied Physics, Military University of Technology, 2 Kaliskiego Str., Warsaw 00-908, Poland \\ ${ }^{5}$ Helmholtz-Zentrum Berlin für Materialien und Energie, M-I1,1 Hahn Meitner Platz, Berlin 14109, Germany \\ ${ }^{6}$ Chemistry Department, Warsaw University, 1 Pasteura Str., Warsaw 02 093, Poland
}

Received December 10, 2010, revised February 21, 2011

\begin{abstract}
The magnetic investigations of potassium holmium double tungstate $\mathrm{KHo}\left(\mathrm{WO}_{4}\right)_{2}$ have been performed. The results of measurements of magnetic susceptibility and magnetization as a function of temperature ( $T$ from $0.3 \mathrm{~K}$ up to $100 \mathrm{~K}$ ) and magnetic field (up to $1.5 \mathrm{~T}$ ) are presented. A strong anisotropy of magnetic properties was found. The magnetic measurements data were used to calculate the interaction energy. It was shown that the interactions between nearest neighbors $\mathrm{Ho}^{3+}$ ions have antiferromagnetic character.

PACS: $75.30 . \mathrm{Gw}$ Magnetic anisotropy;

75.30.Cr Magnetic susceptibility;

75.10.Dg Crystal-field theory and spin Hamiltonians.
\end{abstract}

Keywords: rare earth double tungstate, magnetic susceptibility, magnetization, anisotropy.

\section{Introduction}

The most extensively studied rare-earth (RE) double tungstates are crystals containing Dy and Er, in which the lowest energy levels are Kramer's doublets. The magnetism of RE double tungstates results from competition between spin-spin, dipole-dipole and magnetoelastic interactions. A very low temperature phase transition to antiferromagnetic (AF) state is the characteristic property of these low-symmetry systems and indicates that the exchange interactions are weak. For example, the Néel temperature $T_{N}$ is equal to $0.6,0.7,0.82$ and $0.85 \mathrm{~K}$ for $\mathrm{KDy}\left(\mathrm{WO}_{4}\right)_{2}, \mathrm{KTb}\left(\mathrm{WO}_{4}\right)_{2}, \mathrm{RbDy}\left(\mathrm{WO}_{4}\right)_{2}$ and $\mathrm{KEr}\left(\mathrm{WO}_{4}\right)_{2}$, respectively [1-3].
Mainly, the optical properties were studied for the double tungstates doped with holmium. So far, in the double tungstates $\mathrm{KLu}\left(\mathrm{WO}_{4}\right)_{2}$ and $\mathrm{KGd}\left(\mathrm{WO}_{4}\right)_{2}$ doped with holmium ions the laser emission was observed [4,5]. The results of spectroscopic studies of $\mathrm{KHo}\left(\mathrm{WO}_{4}\right)_{2}, \mathrm{KLu}\left(\mathrm{WO}_{4}\right)_{2}$ and $\mathrm{KGd}\left(\mathrm{WO}_{4}\right)_{2}: \mathrm{Ho}^{3+}$ were presented in Refs. 6-8.

In the $\mathrm{KHo}\left(\mathrm{WO}_{4}\right)_{2}$ crystals under influence of the low symmetry crystal field all the energy levels of the $\mathrm{Ho}^{3+}$ ions are singlets, in contrary to $\mathrm{Dy}^{3+}, \mathrm{Er}^{3+}$ and $\mathrm{Nd}^{3+}$ ions, where energy levels are dublets in the same low symmetry crystal field [9].

It should be noted that magnetic properties of the $\mathrm{KHo}\left(\mathrm{WO}_{4}\right)_{2}$ crystal have been studied very rarely. There is only one paper [10], which describes the magnetic experi- 
mental results obtained along the crystallographic axes of potassium holmium double tungstate.

In this paper, the results of magnetic measurements and theoretical description of potassium-holmium double tungstate are presented. As a result, the peculiarities of magnetic behavior of $\mathrm{KHo}\left(\mathrm{WO}_{4}\right)_{2}$ are determined.

\section{Experimental}

Magnetic properties of $\mathrm{KHo}\left(\mathrm{WO}_{4}\right)_{2}$ compound were investigated in the range of temperatures between 0.5 and $140 \mathrm{~K}$ in magnetic fields up to $2 \mathrm{~T}$ by means of a vibrating magnetometer method. Temperature dependences of principal values of a magnetic susceptibility was measured in a temperature range $0.5-100 \mathrm{~K}$. Temperatures down to $1.8 \mathrm{~K}$ were obtained by pumping of $\mathrm{He}^{4}$, and even lower temperatures down to $0.5 \mathrm{~K}$ by pumping of $\mathrm{He}^{3}$.

The magnetization measurements for the $\mathrm{KHo}\left(\mathrm{WO}_{4}\right)_{2}$ double tungstate along the magnetic axes $\mathbf{x}, \mathbf{y}$ and $\mathbf{z}$ have been carried out using the vibrating sample magnetometer (PAR Model 4500) over the temperature range 5-60 K and in magnetic field up to $1.5 \mathrm{~T}$. An angular dependences of magnetization allow to determine the magnetic $\mathbf{x}, \mathbf{y}, \mathbf{z}$ axes, among which the $\mathbf{y}$ axis coincides with the crystalline $\mathbf{b}$ axis and the $\mathbf{x}$ and $\mathbf{z}$ axes lay in the $a c$ plane. The magnetic $\mathbf{z}$ and $\mathbf{x}$ axes correspond to the directions of maximal and minimal values of magnetization in $a c$ plane, respectively. The angle between the $c$ and $z$ axes is of $15^{\circ}$ and the $a$ axis is set at an angle of $55^{\circ}$ to the $x$ axis.

\section{Magnetic properties of singlet magnet $\mathrm{KHo}\left(\mathrm{WO}_{4}\right)_{2}$}

The temperature dependences of differential magnetic susceptibility and magnetization are presented in Figs. 1-3. The anisotropy of susceptibility was observed over the

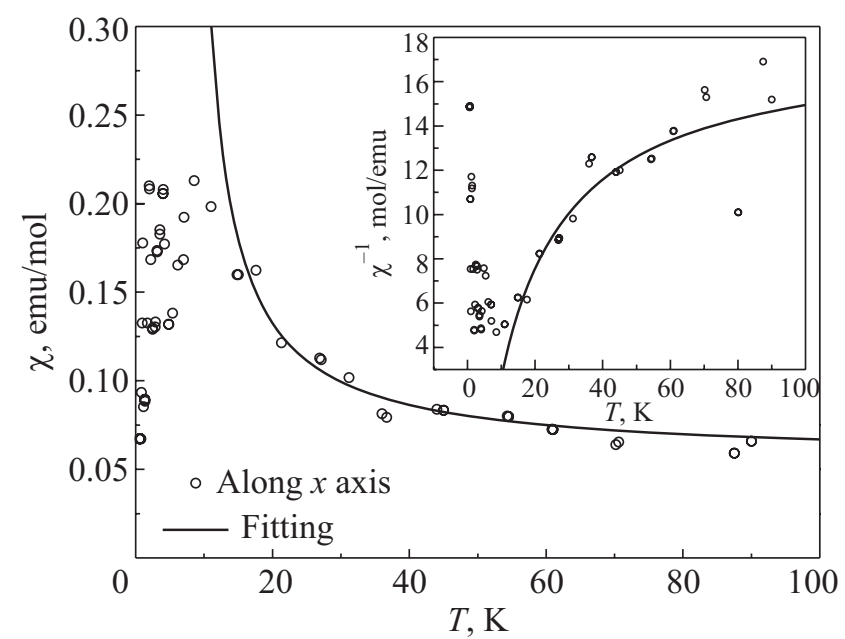

Fig. 1. Temperature dependence of the magnetic susceptibility and inverse magnetic susceptibility (inset) compared with CurieWeiss law dependence (lines) for the $x$ direction in magnetic field of $0.1 \mathrm{~T}$ in temperature range of $0.6-100 \mathrm{~K}$. The high temperature fitting dependences is described by Eq. (1). whole temperature interval. The presence of a strong anisotropy is confirmed by the magnetization data and is due to the influence of a low symmetry crystalline field acting on the $\mathrm{Ho}^{3+}$ ions. In magnets with energy singlets, an unusual magnetic behavior is expected and it is difficult to predict the characteristics of magnetic ordering. The criterion for the appearance of a magnetically ordered state in these materials within two-level singlet-singlet approximation is the fulfillment of the relation $|I|>\delta$, where $I$ is the exchange integral and $\delta$ is the energy gap. In $\mathrm{KHo}\left(\mathrm{WO}_{4}\right)_{2}$, the distance between the two lowest singlets and higher energy levels exceeds by far $\delta$. Both the magnetic susceptibility and magnetization do not demonstrate any magnetic ordering for temperature higher than $1.5 \mathrm{~K}$. This suggests that the condition $|I|>\delta$ is not fulfilled. At $T \sim 20 \mathrm{~K}$, the lines, which describe temperature dependences of susceptibility along the a and c directions, change the angle of inclination. This may be connected with structural phase transition at $T=5.5 \mathrm{~K}$ [11].

The temperature dependences of the magnetic susceptibility along the $x, y$ and $z$ directions (Figs. 1-3) were analyzed in temperature interval from 20 to $130 \mathrm{~K}$. The fitting for experimental points was made using a modified CurieWeiss law:

$$
\chi_{i}=\chi_{0 i}+\frac{C_{i}}{T-\theta_{i}} .
$$

The following values for background susceptibility $\chi_{0 i}$ are of $0.0562,-0.0196$ and $0.0759 \mathrm{emu} /(\mathrm{mol})$ along the $x, y$ and $z$ axes, respectively, were obtained. The Curie constants $C_{i}$ are equal to $0.996,30.2$ and $19.9 \mathrm{emu} \cdot \mathrm{K} / \mathrm{mol}$ and the paramagnetic Curie temperatures $\theta_{i}$ have values of $6.95,-5.91$ and $-16.32 \mathrm{~K}$ along the $x, y$ and $z$ directions, respectively.

The effective numbers of Bohr magneton

$$
n_{i}=\sqrt{\frac{3 k_{B} C_{i}}{\mu_{B}^{2} N_{A}}}
$$

are equal to $2.82,15.55$ and 12.62 along the $x, y$ and $z$ directions, respectively, where $k_{B}$ is the Boltzmann constant, $\mu_{B}$ is the Bohr magneton, and $N_{A}$ is the Avogadro number. The ground state of free $\mathrm{Ho}^{3+}$ ion is ${ }^{5} \mathrm{I}_{8}$. In this case, the quantum theory gives $n_{i}=10.6$ at $J=8$, where $J$ is the total angular momentum.

\section{Theoretical models}

The magnetic susceptibility $\chi_{i}(T)$ was analysed in the frame of the Heisenberg model with crystal field Hamiltonian [12]:

$$
\hat{H}=\sum_{i} \hat{H}_{\mathrm{cr}}^{i}-\frac{1}{2} \sum_{i \neq j} J_{i j} \mathbf{J}_{i} \mathbf{J}_{j}+g_{J} \mu_{B} \mathbf{H} \sum_{i} \mathbf{J}_{i},
$$

where $\mathbf{J}$ and $\mathbf{H}$ are the vectors of total angular moment and applied magnetic field, respectively, $g_{J}$ the Landé factor 
and $J_{i j}$ the indirect exchange between nearest neighbours. Crystal field part of Hamiltonian (2) $\hat{H}_{\mathrm{cr}}^{i}$ is written as

$$
\hat{H}_{\mathrm{cr}}=\sum_{k=2}^{6} \sum_{q=0}^{k}\left(B_{q}^{k} O_{k}^{q}+S_{q}^{k} \Omega_{k}^{q}\right)
$$

where the site dependence of operators was excluded for simplicity. The $O_{n}^{m}$ and $\Omega_{n}^{m}$ operators are expressed as a sum of Stevens operators $O_{k}^{ \pm q}(J)$

$$
\left\{\begin{array}{l}
O_{n}^{m}=\frac{1}{2}\left(O_{n}^{+m}+O_{n}^{-m}\right), \\
\Omega_{n}^{m}=\frac{1}{2 i}\left(O_{n}^{+m}-O_{n}^{-m}\right) .
\end{array}\right.
$$

For the $C_{2}$ symmetry of $\mathrm{Ho}^{3+}$ site $\hat{H}_{\mathrm{cr}}$ involves 15 nonzero crystal field parameters because for the $z$-axis parallel to the $C_{2}$ axis the crystal field parameters with the odd $q$ are equal to zero. Following to Ref. 12 we shall restrict consideration only to second order crystal field parameters since higher order terms give no contribution to the high temperature magnetic susceptibility $\chi_{\alpha}(T)$.

Generally

$$
\chi_{\alpha}^{-1}=\left(\chi_{\alpha}^{0}\right)^{-1}-\lambda, \alpha=x, y, z
$$

where $\lambda=\frac{2 J(0)}{g_{J}^{2} \mu_{B}^{2}}$ and $J(0)=\frac{1}{2} \lambda J_{i j}$. For high temperature approximation

$$
\left(\chi_{\alpha}^{0}\right)^{-1}=\frac{T}{\frac{g_{J}^{2} \mu_{B}^{2} J(J+1)}{3}\left(1+\theta_{\alpha}^{\mathrm{cr}} / T\right)} .
$$

The paramagnetic Curie temperature $\theta_{\alpha}$ consists of two contribution [12]:

$$
\theta_{\alpha}=\theta_{\alpha}^{\mathrm{cr}}+\frac{72 \lambda g_{J}^{2} \mu_{B}^{2}}{3}
$$

It may be shown that $S_{2}^{2} \Omega_{2}^{2}$ term gives no contribution to $\chi_{\alpha}^{0}$, therefore [12]:

$$
\begin{aligned}
& \theta_{z}^{\mathrm{cr}}=-57 B_{2}^{0}, \\
& \theta_{x}^{\mathrm{cr}}=\frac{57}{2}\left(B_{2}^{0}-B_{2}^{2}\right), \\
& \theta_{y}^{\mathrm{cr}}=\frac{57}{2}\left(B_{2}^{0}+B_{2}^{2}\right) .
\end{aligned}
$$

The first and second terms in Eq. (8) are due to crystal field and exchange interactions, respectively. Since [12],

$$
\theta_{x}+\theta_{y}+\theta_{z}=144 J(0)
$$

one can determine the isotropic exchange parameter $J(0)$ using the measured values of the paramagnetic Curie temperature. The experiment gives $\theta_{x}+\theta_{y}+\theta_{z}=-15.27 \mathrm{~K}$. Using Eq. (9) we have $J(0)=-0.106 \mathrm{~K}$. Then the crystal field parameters (see Eq. (7)) are equal to $B_{2}^{0}=0.014 \mathrm{~K}$ and $B_{2}^{2}=-0.408 \mathrm{~K}$.

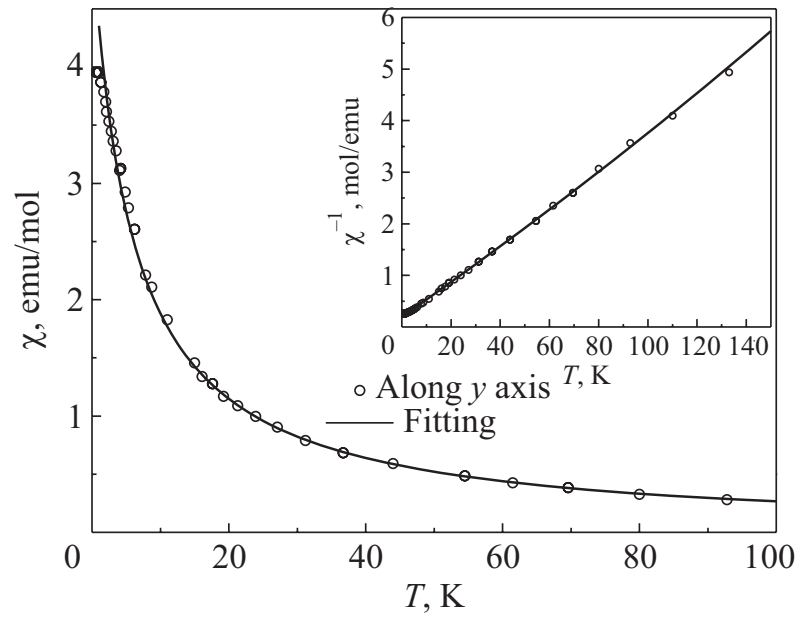

Fig. 2. Temperature dependence of the magnetic susceptibility and inverse magnetic susceptibility (inset) compared with CurieWeiss law dependence (lines) for the $y$ direction in magnetic field of $0.1 \mathrm{~T}$ in temperature range of $0.6-100 \mathrm{~K}$. The high temperature fitting dependences is described by Eq. (1).

The results of fitting to the Curie-Weiss law along the $z$ direction is displayed in Fig. 3. The values of both the paramagnetic Curie temperature and the Curie constant change below $20 \mathrm{~K}$, namely, $\theta_{c}=-2.61 \mathrm{~K}, C_{c}=$ $=14.72 \mathrm{emu} \cdot \mathrm{K} / \mathrm{mol}$.

Unlike the $\chi_{y}(T)$ dependence along the $y$ axis (Fig. 2), the temperature dependence of magnetic susceptibility along $z$ axis deviates from the Curie-Weiss behavior in the low temperature region (from 0 to $3 \mathrm{~K}$ ) (Fig. 3), and above expressions for $\chi_{\alpha}$ can not be used. In this case, all 15 nonzero crystal field parameters should be taken into account. In the case of both low symmetry crystal field and nonKramers ions, the magnetic susceptibility can be calculated in a frame of the singlet-singlet approximation. This is in contrast to the optical and specific heat data and suggests

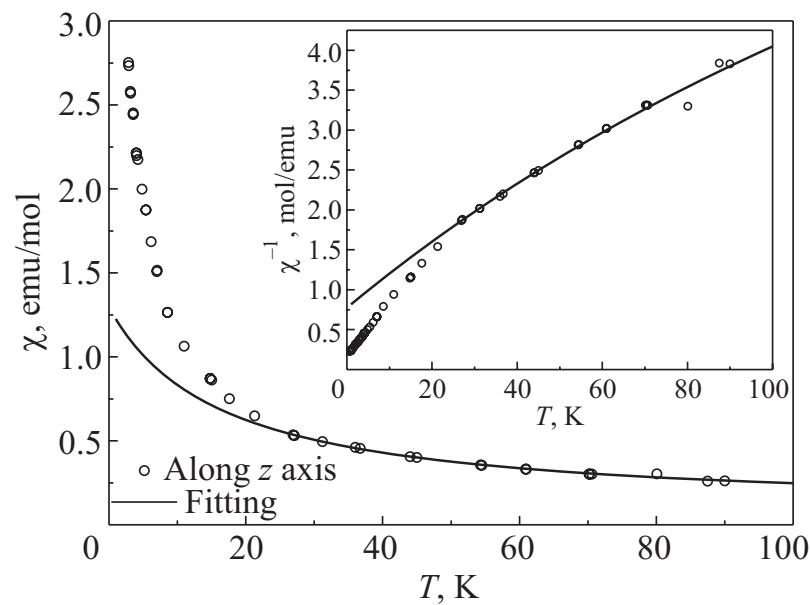

Fig. 3. Temperature dependence of the magnetic susceptibility and inverse magnetic susceptibility (inset) compared with CurieWeiss law dependence (lines) for the $z$ direction in magnetic field of $0.1 \mathrm{~T}$ in temperature range of $0.6-100 \mathrm{~K}$. The high temperature fitting dependences is described by Eq. (1). 
that the two lowest singlets in $\mathrm{KHo}\left(\mathrm{WO}_{4}\right)_{2}$ are not isolated from other crystal field levels. Such situation was observed for $\mathrm{KHo}\left(\mathrm{WO}_{4}\right)_{2}$ and for $\mathrm{Ho}^{3+}$ ion in $\mathrm{KGd}\left(\mathrm{WO}_{4}\right)_{2}[6,8]$.

To describe the low temperature behavior of magnetic susceptibility $\chi_{\alpha}(T)$ we consider the singlet-singlet-singlet model. Apparently, this model should be more exact at temperatures up to $3.5 \mathrm{~K}$ since the energy area of the three lower levels of the holmium ion does not exceed $11 \mathrm{~cm}^{-1}$ [6]. Also, we can not exclude an influence of the indirect exchange of the rare earth ions on location of these singlets. The problem is quite similar to one to be considered by Moriya [13] for the system with competing single ion anisotropy, rhombic distortion and indirect exchange. One can use an effective spin of this system $S=1$. The most general form of the effective spin Hamiltonian with $C_{2}$ symmetry may be written as:

$$
\hat{H}=-D\left(S^{z}\right)^{2}+\frac{\Delta}{2}\left[\left(S^{x}\right)^{2}-\left(S^{y}\right)^{2}\right]-\mu_{B} \sum_{i}^{3} g_{i} H_{i} S^{i} .
$$

In the absence of the applied field with $D<0$ and $\Delta / 2<0$ the energy levels may be presented in series as:

$$
\varepsilon_{0}=0, \varepsilon_{1}=D-\frac{\Delta}{2} \text { and } \varepsilon_{2}=-\Delta .
$$

The longitudinal susceptibility (along the $z$ axis) has the form

$$
\chi_{\|}(T)=\frac{\mu_{B}^{2} g_{z}^{2} N_{A}}{k_{B}} \frac{\phi_{\|}^{c}(T)}{1-2 J_{z}(0) \phi_{\|}^{c}(T)}
$$

where the generalized susceptibility $\phi_{\|}^{c}(T)$ is equal:

$$
\phi_{\|}^{c}=\frac{2}{\Delta} \frac{1-\exp (-\beta \Delta)}{1+\exp (-\beta \Delta)+\exp \left(-\beta\left(D+\frac{\Delta}{2}\right)\right)} .
$$

Here $\beta=1 / T$. Similarly, for transversal susceptibility (easy plane) we have

$$
\chi_{\perp}(T)=\frac{\mu_{B}^{2} g_{x}^{2} N_{A}}{k_{B}} \frac{\phi_{\perp}^{c}(T)}{1-2 J_{x}(0) \phi_{\perp}^{c}(T)},
$$

where the generalized susceptibility $\phi_{\perp}^{c}(T)$ is equal:

$$
\phi_{\perp}^{c}(T)=\frac{2}{\left(D-\frac{\Delta}{2}\right)} \frac{\exp (-\beta \Delta)-\exp \left(-\beta\left(D+\frac{\Delta}{2}\right)\right)}{1+\exp (-\beta \Delta)+\exp \left(-\beta\left(D+\frac{\Delta}{2}\right)\right)} \text {. }
$$

In the geometry of an "easy" plane $(D<0)$ at zero rhombic distortion $\Delta=0$, the criterion of Moriya [9] is realized: at $D / J(0)<-4$ the magnetic ordering in system is absent. In Fig. 4 the Curie temperature $T_{C}$ as function of $D$ (in $J(0)$ units) is presented (see curve 1 ). One can see a realization of Moriya's criterion for singlet model. For $\Delta \neq 0$, the situation is more complicated. For the negative $\Delta$, the Curie

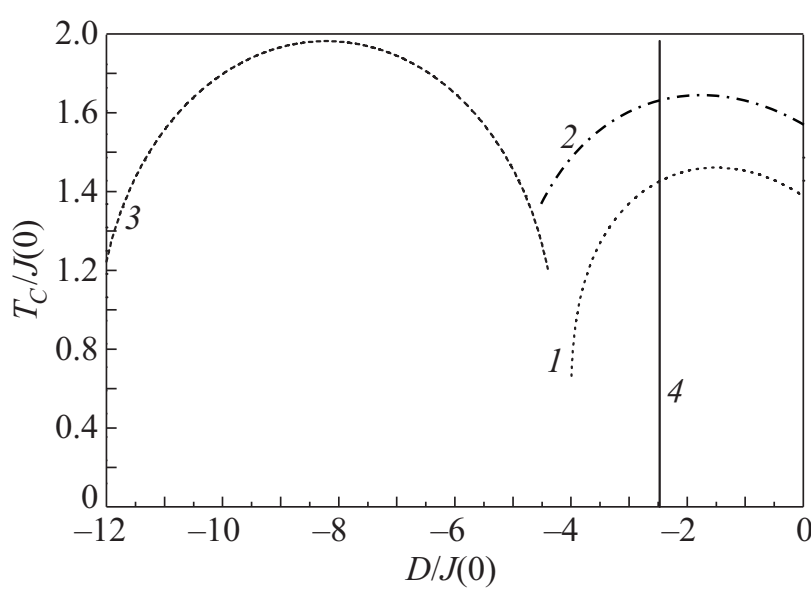

Fig. 4. The Curie temperature $T_{C}$ as a function of $D$ (in $J(0)$ units) is presented. The dot line (1) conforms isotropic Moriya model $(\Delta=0)$, dash-dot line (2) and dash line (3) present dependence $T_{C}$ for case $\Delta \neq 0$ of the following parameters: $D /\left|J_{y}(0)\right|=-0.33$ and $\Delta /\left|J_{y}(0)\right|=-2.17$, or $D /\left|J_{z}(0)\right|=-2.47$ and $\Delta /\left|J_{z}(0)\right|=-16.42$. The solid line (4) shows the dependence for the value parameter $D /\left|J_{z}(0)\right|=-2.47$.

temperature deviates from Moriya dependences and at large module $\Delta$ values the magnetic order is destroyed already at small module $D$ in contrast with Moriya prediction (see curve 1 in Fig. 4). Probably, such a situation is realized in $\mathrm{KHo}\left(\mathrm{WO}_{4}\right)_{2}$.

The temperature dependences of the magnetic susceptibility and fitting curves using Eqs. (12) and (14) of the three magnetic axes are presented in Figs. 5. and 6. The inset of Fig. 5 present these dependences at low temperatures. The anisotropy of the magnetic susceptibility was observed over the whole temperature interval. Indeed, in

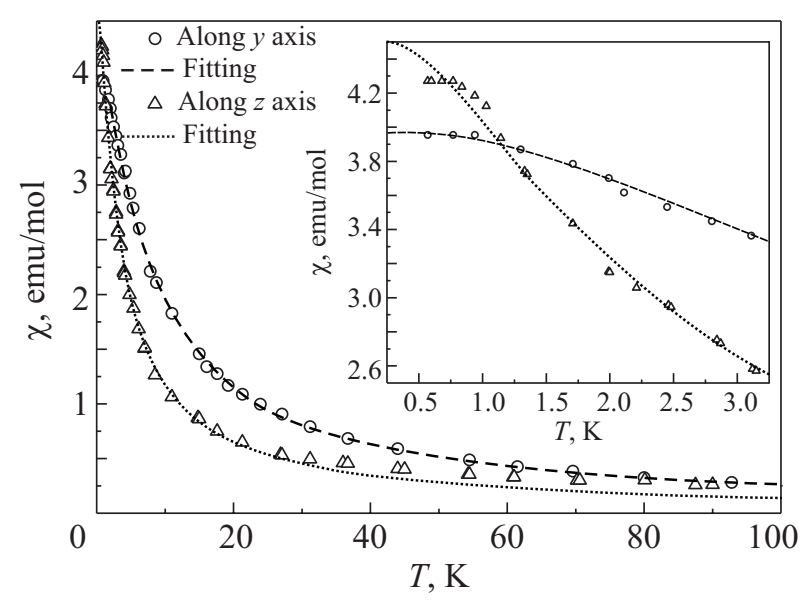

Fig. 5. Temperature dependence of the magnetic susceptibility $\chi_{i}$ (points) and low temperature fitting (line) for the transversal susceptibility along the $y$ and $z$ directions in temperature range of 0.6-100 K. In the inset, the temperature dependence of the magnetic susceptibility $\chi_{i}$ (points) and the best fit in temperature range of $0.6-3 \mathrm{~K}$ are presented. 


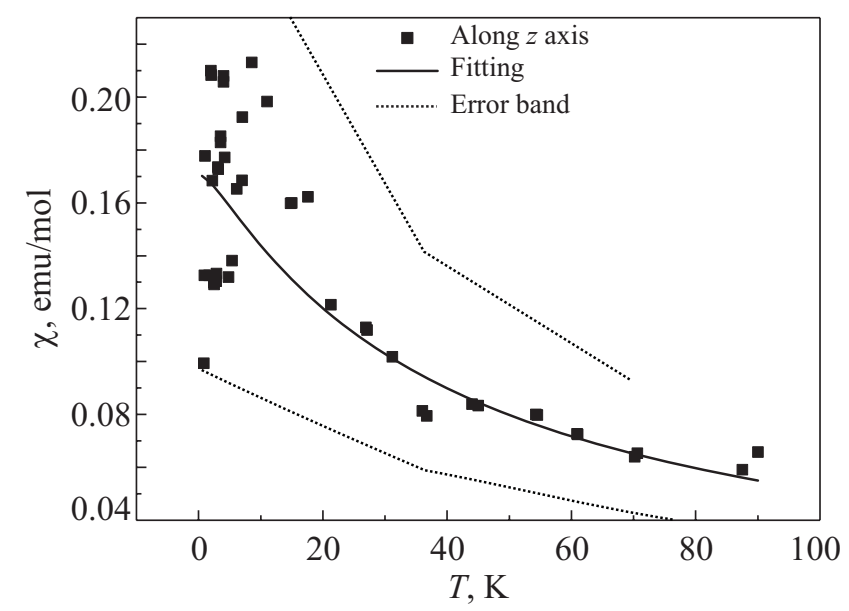

Fig. 6. Temperature dependence of the magnetic susceptibility $\chi_{i}$ (points) and low temperature fitting (line) for the longitudinal component (the $x$ direction) in temperature range of 0.6-100 K. The area between dot lines presents fitting error band.

Fig. 5 the fitting curve using Eq. (14) to experimental temperature dependence of the magnetic susceptibility along the $y$ and $z$ axes is presented. We obtain a good correspondence between the experiment and theory in a wide temperature interval. The results suggest an easy plane type magnetic anisotropy since $D=-1.41 \mathrm{~K}<0$ and a big rhombic distortion in easy plane: $\Delta=-9.362 \mathrm{~K}$. Within the easy plane we have the values of the exchange parameter $J_{y}(0)=-4.3 \mathrm{~K}$ and $J_{z}(0)=-0.57 \mathrm{~K}$, a large $g$-factor equal 16.95 and 10.9, respectively, for the $y$ and $z$ axes. In accordance with Eq. (10) at $H_{i}=0$, we obtain energy levels at 0 , 2.43 and $6.88 \mathrm{~cm}^{-1}$. From this result one can conclude that not only parameters $B_{2}^{0}$ and $B_{2}^{2}$ determine the energy levels of the Hamiltonian (2).

The axis $x$ is supposed to be along the hard direction. The small value of the susceptibility $\chi_{x}$ does not allow to make an exact fitting along this direction. In Fig. 6 the fitting curve (12) to experimental temperature dependence of the susceptibility along $z$ axis is presented. The error of the fitting is high and reach about $30 \%$.

Now we can consider the possibility of the absence of magnetic ordering in $\mathrm{KHo}\left(\mathrm{WO}_{4}\right)_{2}$. Then we have following relations $D /\left|J_{y}(0)\right|=-0.33, \Delta /\left|J_{y}(0)\right|=-2.17, D /\left|J_{z}(0)\right|=$ $=-2.47, \Delta /\left|J_{z}(0)\right|=-16.42$. For this parameters the theoretical dependences $T_{C} / J(0)$ from $D /\left|J_{i}(0)\right|$ in Fig. 4 was calculated. The Moriya model was developed for isotropic model. For $\mathrm{KHo}\left(\mathrm{WO}_{4}\right)_{2}$ single crystal anisotropic magnetic properties were observed and this results in various exchange parameters. Two theoretical dependences $T_{C} / J(0)$ were shown in Fig. 4. The curve for the $y$ direction suggests $T_{C}$ less then $7.8 \mathrm{~K}$ and for the $z$ direction $T_{C}$ smaller $0.23 \mathrm{~K}$. From Fig. 4 we can see that for $D /\left|J_{i}(0)\right|>-3.7$ and the magnetic ordering in system is absent. For $\mathrm{KHo}\left(\mathrm{WO}_{4}\right)_{2}$ we obtain $D /\left|J_{z}(0)\right|=-2.47>-3.7$, i.e., magnetic order- ing does not appear. The $D /|J(0)|$ is close to the critical value -3.7 . In this situation the applied magnetic field can induce various magnetic phase transitions depending on a geometry when the field will compete with anisotropy and exchange.

These results coincide with the results of the neutron scattering research, in which the spontaneous magnetic phase transition was not observed up to $37 \mathrm{mK}[14,15]$. However, the neutron investigations show an existence of the induced magnetic phase transition. At least one antiferromagnetic component exists at field $H=0.1 \mathrm{~T}$, but the complex of magnetic phase transition with antiferroferromagnetic character was observed at $H=1 \mathrm{~T}[14,15]$.

The $M(H)$ dependences for the $x$ and $y$ axes are typical for paramagnetic materials. We want to attract attention on the behavior of magnetic field dependence of magnetization along the $z$ axis. It is necessary to stress that experimental $M(H)$ curves demonstrate an unusual hysteresis without coercive force and remanence magnetization [10].

\section{Conclusion}

Summarizing, the parameter of exchange interactions between nearest neighbors $\mathrm{Ho}^{3+}$ ions was estimated. The negative sign of exchange parameter indicates on antiferromagnetic character of the exchange interaction. A reasonable agreement between experimental and calculated values of energy level splitting of the lowest singlets was obtained. The $D /|J(0)|$ is close to the critical value -3.7 and in this situation the applied magnetic field can induce different magnetic phases depending on a geometry. The field competes in this case with anisotropy and exchange interaction. This fact can interpret anomalous behavior of the magnetic susceptibility at $0.6 \mathrm{~K}$.

\section{Acknowledgements}

This work was supported by EU project DT-CRYS, NMP3-CT-2003-505580, by Polish State Committee on Science (KBN) (decision of project No. 72/E-67/SPB/6. PR/DIE 430/2004-2006). The neutron diffraction measurements at BENSC was carried out along the proposals PHY-01-1254, -1334 and PHY-01-1441.

1. M.T. Borowiec, V.P. Dyakonov, A. Jedrzejczak, V. Markovich, A. Pavlyuk, H. Szymczak, E. Zubov, and M. Zaleski, J. Low Temp. Phys. 110, 1003 (1998); M.T. Borowiec, V.P. Dyakonov, A. Jedrzejczak, V. Markovich, A. Pavlyuk, H. Szymczak, E. Zubov, and M. Zaleski, Phys. Lett. A243, 85 (1998).

2. M.T. Borowiec, V.P. Dyakonov, K. Wozniak, Ł. Dobrzycki, M. Berkowski, E.E. Zubov, E. Michalski, A. Szewczyk, M.U. Gutowska, T. Zayarnyuk, and H. Szymczak, J. Phys.: Condens. Matter 19, 056206 (2007).

3. E. Khatsko, A. Loginov, A. Cherny, and A.I. Rykova, Physica B378-380, 1126 (2006). 
4. E.V. Zharikov, C. Zaldo, and F. Diaz, MRS Bulletin 34, 271 (2009).

5. V. Jambunathan, X. Mateos, M.C. Pujol, J.J. Carvajal, J. Massons, M. Aguiloacute, and F. Deaz, J. Lumin. 129, 1882 (2009).

6. M.T. Borowiec, A. Watterich, T. Zayarnyuk, V.P. Dyakonov, A. Majchrowski, J. Zmija, M. Baranski, and H. Szymczak, J. Appl. Spectros. 71, 888 (2004); L. Kovacs, M.T. Borowiec, A. Majchrowski, A. Baraldi, and R. Capelletti, Cryst. Res. Tech. 40, 444 (2005).

7. L. Macalik, P.J. Deren, J. Hanuza, W. Strek, A.A. Demidovich, and A.N. Kuzmin, J. Mol. Struct. 450, 179 (1998).

8. M.C. Pujol, J. Massons, M. Aguilo, F. Diaz, M. Rico, and C. Zaldo, IEEE J. Quantum Electron. 38, 93 (2002); M.C. Pujol, C. Cascales, M. Rico, J. Massons, F. Diaz, P. Porcher, and C. Zaldo, J. Alloys Comp. 321-325, 321 (2001); D. Kasprowicz, T. Runka, M. Szybowicz, M. Drozdowski, A. Majchrowski, E. Michalski, and J. Zmija, J. Mol. Struct. 792-793, 139 (2006).
9. K.N.R. Taylor and M.I. Darby, Physics of Rare Earth Solids, Chapman and Hall Ltd, London (1992).

10. M.T. Borowiec, E.N. Khatsko, V.P. Dyakonov, A.I. Rykova, A. Majchrowski, T. Zayarnyuk, M. Barański, J. Żmija, and H. Szymczak, Cent. Eur. J. Phys. 9, 260 (2011).

11. M.T. Borowiec, V.P. Dyakonov, A. Szewczyk, M.U. Gutowska, T. Zayarnyuk, E.E. Zubov, A. Majchrowski, E. Michalski, J-U. Hoffmann, K. Prokes, K. Woźniak, Ł. Dobrzycki, E.N. Khatsko, A.I. Rykova, V. Domukhovski, M. Barański, V. Shtyrkhunova, J. Żmija, and H. Szymczak, Phase Transition, in press (2011).

12. A.K. Zvezdin, V.M. Matveev, A.A. Mukhin, and A.J. Popov, Rare Earth Ions in Magnetically Ordered Crystals, Nauka, Moscow (1985).

13. T. Moriya, Phys. Rev. 117, 635 (1960).

14. M.T. Borowiec, J.-U. Hoffmann, and I. Glavatsky, BENSC Exp. Rep. 2003, 13 (2004).

15. M.T. Borowiec, K. Prokes, and V. Sikolenko, BENSC Exp. Rep. 2004, 71 (2005). 